\title{
Alterations in gene expression and DNA methylation profiles in gastric cancer cells obtained from ascitic fluids collected before and after chemotherapy
}

\author{
OSAMU MAEDA ${ }^{1}$, AYUMU MATSUOKA ${ }^{1}$, KAZUHIRO FURUKAWA $^{2}$, RYOJI MIYAHARA ${ }^{2}$, \\ YOSHIKI HIROOKA ${ }^{3}$ and YUICHI ANDO ${ }^{1}$ \\ ${ }^{1}$ Department of Clinical Oncology and Chemotherapy, Nagoya University Hospital, Nagoya, Aichi 466-8560;
${ }^{2}$ Department of Gastroenterology and Hepatology, Nagoya University Graduate School of Medicine, Nagoya, Aichi 466-8550;
${ }^{3}$ Department of Endoscopy, Nagoya University Hospital, Nagoya, Aichi 466-8560, Japan
}

Received November 22, 2018; Accepted April 30, 2019

DOI: $10.3892 /$ mco.2019.1858

\begin{abstract}
Resistance to anticancer drugs is a critical issue in cancer treatment. Alterations in gene expression and DNA methylation profiles that accompany the acquisition of drug resistance are associated with resistance mechanisms. To analyze chemotherapy-associated alterations in gene expression and DNA methylation in gastric cancer cells obtained from ascites, ascitic fluids were collected from a patient with gastric cancer before chemotherapy with capecitabine and oxaliplatin (CapeOX), and after the disease had progressed. The fluids were cultured for 10 days, passaged into new flasks, and cultured for an additional 2 weeks. Normal cells, including white blood cells and mesothelial cells, were removed. The expression and DNA methylation profiles of 18,185 genes were analyzed using microarray, and compared between cells in ascitic fluids collected before and after the chemotherapy with CapeOX. In addition, fluorouracil- and oxaliplatin-resistant AGS cells were established and analyzed. Pathways having genes with expression profiles altered by CapeOX included those associated with 'signaling by G-protein-coupled receptor' and the 'immune system'. Genes that were commonly expressed at higher levels in CapeOX-resistant ascitic cells, fluorouracil-resistant AGS cells. and oxaliplatin-resistant AGS cells compared with those in untreated cells included telomerase reverse transcriptase (TERT), apolipoprotein C1 (APOC1) and serine/threonine/tyrosine kinase 1 (STYK1), whereas genes commonly expressed at lower levels in the three drug-resistant cell types compared with the untreated cells included defensin $\beta 4 \mathrm{~A}$ (DEFB4A). A comparatively large number of
\end{abstract}

Correspondence to: Dr Osamu Maeda, Department of Clinical Oncology and Chemotherapy, Nagoya University Hospital, 65 Tsurumai-cho, Showa-ku, Nagoya, Aichi 466-8560, Japan E-mail: maeda-o@med.nagoya-u.ac.jp

Key words: gastric cancer, malignant ascites, fluorouracil, oxaliplatin, capecitabine, drug resistance genes exhibited altered methylation levels in drug-resistant AGS cells compared with the CapeOX-resistant cells. In addition, among the genes expressed at higher levels in decitabine-treated AGS cells, the majority were expressed at higher levels in fluorouracil-resistant AGS cells, and exhibited lower methylation levels. Taken together, the present study has demonstrated that comparing the expression profiles of gastric cancer cells obtained from ascitic fluids before and after chemotherapy with the expression profiles of drug-resistant cultured cells is a useful method for analyzing the molecular mechanisms underlying chemotherapy resistance.

\section{Introduction}

The standard treatment for unresectable gastric cancer is combination chemotherapy using fluoropyrimidine and platinum agents (1-3). The fluoropyrimidine agents fluorouracil, capecitabine and tegafur/gimeracil/oteracil (S-1), and the platinum agents cisplatin and oxaliplatin, are used in the treatment of gastric cancer. Although chemotherapeutic regimens are selected based on the results of clinical trials, the effectiveness of treatment regimens varies among patients. In cancer cells, drug resistance to chemotherapeutic agents is an important problem that results in ineffective treatment. For some molecular-targeted agents, there are useful predictive markers, such as epidermal growth factor receptor (EGFR) mutation, RAS mutation and HER2 amplification. However, for the majority of cytotoxic drugs, few biomarkers are available that are able to predict the sensitivity and resistance of the cancer cells to chemotherapeutic agents. Although the acquisition of drug resistance is associated with alterations in the functions of molecules and signaling pathways (4), the mechanisms underlying resistance have yet to be fully elucidated.

Malignant ascites due to the peritoneal dissemination of advanced cancer, including gastric cancer, causes distressing symptoms, including abdominal fullness and appetite loss. When chemotherapy is effective, the volume of malignant ascites is decreased, causing symptoms to improve. Although diuretics are used to relieve certain of the symptoms, their effects are often insufficient, and they can cause adverse 
effects, including dehydration and electrolyte abnormalities. Paracentesis is also performed to decrease abdominal fullness, although repeated procedures are often necessary since its effects are not long-lasting (5). The drained ascites contain malignant cells mixed with normal cells, including blood and mesothelial cells. In the present study, malignant cells were extracted from malignant ascites, and the biological features of these cells were analyzed with the expectation that important information concerning drug resistance would be revealed.

Gene alterations observed following the acquisition of drug resistance could be associated with the mechanisms underlying drug resistance; therefore, improving our understanding of these alterations may provide us with biomarkers that could be used to ensure selection of appropriate treatments. In the present study, early passage cells, that were passaged only a few times, were used. The cells were derived from malignant ascites collected from a patient with gastric cancer. Alterations in the gene expression and DNA methylation profiles of cancer cells obtained from the ascites were analyzed, which was repeatedly collected during the clinical course of chemotherapy.

\section{Materials and methods}

Patient. A 56-year-old man was diagnosed with gastric cancer with peritoneal dissemination, who underwent chemotherapy with capecitabine and oxaliplatin [CapeOX; specifically, capecitabine $\left(2,000 \mathrm{mg} / \mathrm{m}^{2} /\right.$ day $)$ was administered on days $1-14$, and oxaliplatin $\left(130 \mathrm{mg} / \mathrm{m}^{2}\right)$ was given on day 1 every 3 weeks]. After two cycles of CapeOX, computed tomography revealed that the para-aortic lymph nodes were reduced in size; therefore, CapeOX treatment was judged to have been effective. However, after three courses of CapeOX, the ascites had become uncontrollable, and the disease was judged to be refractory to CapeOX. Since the patient had abdominal distention with massive ascites, repeated paracentesis was necessary to relieve his symptoms.

Ethics approval and consent to participate. The present study was conducted in accordance with the principles of the Declaration of Helsinki and all local regulations. Written informed consent was obtained from the patients. The study protocol was approved by the institutional ethics committee of Nagoya University Hospital.

Culture of ascitic fluid cells and cell lines. Ascitic fluid was drained, and cells were collected prior to CapeOX initiation, immediately before the third cycle of CapeOX, and after completion of the third cycle of CapeOX. Aliquots of ascitic fluid $(40 \mathrm{ml})$ were centrifuged at $190 \mathrm{x}$ g for $10 \mathrm{~min}$ at $4^{\circ} \mathrm{C}$, and the resulting cell pellets were resuspended in RPMI-1640 (Thermo Fisher Scientific, Inc.) supplemented with $10 \%$ fetal bovine serum (Equitech-Bio) and $1 \mathrm{X}$ antibiotic-antimycotic (Thermo Fisher Scientific, Inc.) at $37^{\circ} \mathrm{C}$. The cells were subsequently plated in $75-\mathrm{cm}^{2}$ culture flasks and incubated in a humidified incubator containing $5 \% \mathrm{CO}_{2}$ for 10 days. The culture medium was exchanged twice a week. Subconfluent cells were subsequently passaged to new flasks at a 1:4 ratio, and cultured for an additional 2 weeks. It was assumed that a small percentage of normal cells, including mesothelial cells and white blood cells, would be able to survive under culture conditions that are not optimized for non-cancerous cells. However, the morphology of the cultured cells was checked to confirm that the majority of the cultured cells were cancer cells.

AGS gastric cancer cells were purchased from the American Type Tissue Culture Collection (ATCC). In experiments involving treatment with the demethylating agent, decitabine (also known as 5-aza-dC), cells were seeded on day 0 and subsequently exposed to freshly prepared $10 \mu \mathrm{mol} / 15$-aza-dC (Sigma-Aldrich; Merck KGaA) for $24 \mathrm{~h}$ on days 1 and 3. After each treatment, the cells were placed in fresh medium, and cells were harvested on day 4 .

Drug-resistant AGS cells. Oxaliplatin-resistant AGS cells were generated by continuously exposing AGC cells to increasing concentrations of oxaliplatin for 5 months. Cell viability was measured by analyzing MTS-formazan reduction using a CellTiter $96^{\circledR}$ AQueous One Solution Cell Proliferation assay (Promega Corporation). AGS cells $\left(2 \times 10^{3}\right)$ were cultured at $37^{\circ} \mathrm{C}$ in 96 -well microplates for $24 \mathrm{~h}$, and subsequently exposed to various concentrations of oxaliplatin for $48 \mathrm{~h}$ to calculate the $\mathrm{IC}_{50}$ of oxaliplatin (i.e., the concentration required to give half-maximal inhibition). Cells with 5-fluorouracil resistance were previously established, as reported elsewhere (4).

Extraction of DNA and RNA from cultured cells. Cultured cells were grown to subconfluence, collected and stored at $-80^{\circ} \mathrm{C}$ for DNA extraction; Ambion ${ }^{\circledR}$ RNAlater tissue storage reagent (Thermo Fisher Scientific, Inc.) was used to store the cells in advance of RNA extraction. To extract DNA and RNA, DNA Mini kits and RNA Mini kits (both from Qiagen) were used, respectively.

Gene expression analysis using a microarray. Expression analysis was performed using a SurePrint G3 Human GE 8x60K microarray (Agilent Technologies, Inc.). A >2-fold difference in signal intensity was judged to be significant (4). The expression level in cells before treatment with anticancer drugs was set arbitrarily to one, in order that the relative expression levels in cells after treatment could be calculated.

DNA methylation microarray. Bisulfite-converted DNA was used for hybridization on an Infinium HumanMethylation450 BeadChip (Illumina, Inc.). The $\beta$-value [calculated as follows: intensity of the methylated allele $(\mathrm{M}) /($ intensity of the unmethylated allele (U) + intensity of the methylated allele $(M)+100)$ ] was calculated for each $\mathrm{CpG}$ site. Genes exhibiting a difference in $\beta$-values $>0.1$ were extracted.

Pathway analysis. Pathway analysis was performed using TargetMine (http://targetmine.mizuguchilab.org/targetmine/begin.do; National Institute of Biomedical Innovation). Pathways with $\mathrm{P}<0.05$, according to the Benjamini-Hochberg method (6), were judged to be significantly enriched.

Reverse transcription-quantitative polymerase chain reaction $(R T-q P C R)$. cDNA was generated from $8 \mu \mathrm{g}$ RNA using SuperScript ${ }^{\circledR}$ III Reverse Transcriptase (Thermo Fisher Scientific, Inc.). To validate the mRNA expression profiles 
Table I. Pathways associated with genes expressed at lower levels after CapeOX treatment.

\begin{tabular}{lcc}
\hline Pathway & P-value & No. of genes \\
\hline GPCR ligand binding & 0.0001 & 32 \\
Class A/1 (rhodopsin like receptors) & 0.0037 & 23 \\
Signaling by GPCRs & 0.0077 & 52 \\
Peptide ligand binding receptors & 0.0190 & 15 \\
GPCR downstream signaling & 0.0190 & 48 \\
Neuroactive ligand receptor interaction & 0.0190 & 19 \\
NGF independent TRKA activation & 0.0463 & 3 \\
\hline
\end{tabular}

CapeOX, capecitabine and oxaliplatin; GPCR, G protein coupled receptor; NGF, nerve growth factor; TRKA, tropomyosin receptor kinase A.

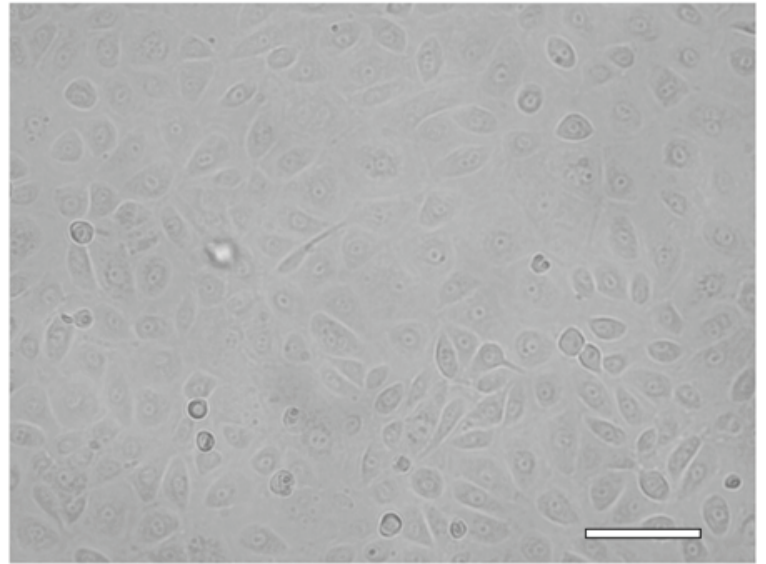

Figure 1. Microscopic photograph of cells obtained from ascitic fluids. The cells were cultured and passaged once (scale bar, $100 \mu \mathrm{m}$ ).

of genes of interest, RT-qPCR was performed using Applied Biosystems $^{\circledR}{ }^{\text {TaqMan }}{ }^{\mathrm{TM}}$ Gene Expression assays and TaqMan ${ }^{\mathrm{TM}}$ Gene Expression Master mix (Thermo Fisher Scientific, Inc.). The assay IDs of the genes defensin $\beta 4 \mathrm{~A}$ (DEFB4A), protocadherin-20 (PCDH20), and GAPDH were Hs00823638_m1, Hs00276707_m1 and Hs02758991_g1, respectively. GAPDH expression was used as the reference, and a serial dilution of cDNA from non-cancerous tissue (qPCR Human Reference cDNA; Takara Bio, Inc.) was set up for the purposes of normalization. The thermal cycler program conditions consisted of an initial step at $50^{\circ} \mathrm{C}$ for $2 \mathrm{~min}$, a denaturation step at $95^{\circ} \mathrm{C}$ for $10 \mathrm{~min}$, and 40 amplification cycles comprising denaturation at $95^{\circ} \mathrm{C}$ for $15 \mathrm{sec}$ and annealing and extension at $60^{\circ} \mathrm{C}$ for $1 \mathrm{~min}$. The expression level in cells before treatment with anticancer drugs was set to one, in order that the relative expression levels in cells after treatment could be calculated.

Statistical analysis. A one-way analysis of variance and a Games-Howell post hoc test were used to compare the levels of gene expression obtained by RT-qPCR. P $<0.05$ was considered to indicate a statistically significant difference.

\section{Results}

Early passage gastric cancer cells from ascitic fluid. Ascitic fluids from gastric cancer were cultured for 10 days, passaged to new flasks, and cultured for an additional 2 weeks. The morphology of cells isolated from ascitic fluids was assessed (Fig. 1), and it was determined that atypical cells had been derived from the gastric cancer cells.

Alterations in the gene expression and DNA methylation profiles of cancer cells in ascitic fluids collected before and after chemotherapy. Cells collected before and after three courses of CapeOX treatment were used to analyze the expression and DNA methylation profiles of 18,185 genes. The expression profiles of 530 of those genes revealed that they were expressed at lower levels after, rather than before, CapeOX, whereas the DNA methylation profiles of 25 genes showed that they were expressed at higher levels after, rather than before, CapeOX (Table SI). Of the 530 genes shown to have a lower level of expression after CapeOX treatment, a pathway analysis revealed that 32 were associated with 'signaling by G-protein-coupled receptor (GPCR)', 15 were associated with 'peptide ligand-binding receptors', and 48 were associated with 'GPCR downstream signaling' (Tables I and SII). Additionally, of those 530 genes, four [nephrin (NPHS1), FXYD domain containing ion transport regulator 1 (FXYD1), WNT1-inducible-signaling pathway protein 1 (WISP1) and flavin-containing monooxygenase 1 (FMO1)] exhibited increased DNA methylation profiles.

On the other hand, the expression profiles of 703 genes revealed that they were expressed at higher levels after CapeOX, and the DNA methylation profiles of four genes showed they were expressed at lower levels after CapeOX (Table SI). Of those 703 genes, a pathway analysis revealed that 134 were associated with the 'immune system', and 52 were associated with 'neutrophil degranulation' (Tables II and SIII). In addition, no genes simultaneously showed an increase in expression and a decrease in DNA methylation among the cells collected before and after CapeOX treatment.

Consistency of gene expression levels between microarray and RT-qPCR analyses. To validate the microarray-based results obtained for gene expression levels, RT-qPCR for two genes, DEFB4A and PCDH20, that were identified to be expressed at lower levels after, rather than before, CapeOX was performed (Fig. 2). A high level of consistency was noted between the results obtained using these two methods. 
Table II. Pathways associated with genes expressed at higher levels after CapeOX treatment.

\begin{tabular}{|c|c|c|}
\hline Pathway & P-value & No. of genes \\
\hline Immune system & $1.63 \mathrm{E}^{-8}$ & 134 \\
\hline Neutrophil degranulation & $1.63 \mathrm{E}^{-8}$ & 52 \\
\hline Staphylococcus aureus infection & $1.63 \mathrm{E}^{-8}$ & 17 \\
\hline Innate immune system & $1.67 \mathrm{E}^{-7}$ & 83 \\
\hline Osteoclast differentiation & $3.42 \mathrm{E}^{-7}$ & 23 \\
\hline Signaling by GPCRs & $5.50 \mathrm{E}^{-7}$ & 88 \\
\hline Immunoregulatory interactions between lymphoid and non lymphoid cells & $1.01 \mathrm{E}^{-6}$ & 22 \\
\hline GPCR downstream signaling & $8.76 \mathrm{E}^{-6}$ & 81 \\
\hline Ga (i) signaling events & $9.29 \mathrm{E}^{-6}$ & 40 \\
\hline Chemokine signaling pathway & $1.25 \mathrm{E}^{-5}$ & 25 \\
\hline Rheumatoid arthritis & $1.25 \mathrm{E}^{-5}$ & 17 \\
\hline Interleukin-10 signaling & $5.47 \mathrm{E}^{-5}$ & 12 \\
\hline Phagosome & $8.19 \mathrm{E}^{-5}$ & 21 \\
\hline Leishmaniasis & $1.45 \mathrm{E}^{-4}$ & 14 \\
\hline CXCR4 mediated signaling events & $2.38 \mathrm{E}^{-4}$ & 16 \\
\hline Extracellular matrix organization & $2.42 \mathrm{E}^{-4}$ & 30 \\
\hline Tuberculosis & $2.54 \mathrm{E}^{-4}$ & 22 \\
\hline Cytokine cytokine receptor interaction & $3.08 \mathrm{E}^{-4}$ & 28 \\
\hline Inflammatory bowel disease & $8.56 \mathrm{E}^{-4}$ & 12 \\
\hline Toxoplasmosis & $9.22 \mathrm{E}^{-4}$ & 16 \\
\hline Chagas disease (American trypanosomiasis) & $1.06 \mathrm{E}^{-3}$ & 15 \\
\hline Class A/1 (Rhodopsin like receptors) & $1.10 \mathrm{E}^{-3}$ & 30 \\
\hline Complement and coagulation cascades & $1.10 \mathrm{E}^{-3}$ & 13 \\
\hline Peptide ligand binding receptors & $1.42 \mathrm{E}^{-3}$ & 21 \\
\hline Intestinal immune network for IgA production & $1.42 \mathrm{E}^{-3}$ & 10 \\
\hline Malaria & $1.42 \mathrm{E}^{-3}$ & 10 \\
\hline Viral myocarditis & $1.42 \mathrm{E}^{-3}$ & 11 \\
\hline Asthma & $1.60 \mathrm{E}^{-3}$ & 8 \\
\hline GPCR ligand binding & $1.68 \mathrm{E}^{-3}$ & 37 \\
\hline Fc $\gamma$ receptor activation & $4.64 \mathrm{E}^{-3}$ & 5 \\
\hline Other semaphorin interactions & $4.82 \mathrm{E}^{-3}$ & 6 \\
\hline Interleukin-8 and CXCR 1 mediated signaling events & $5.92 \mathrm{E}^{-3}$ & 7 \\
\hline Platelet activation & $6.40 \mathrm{E}^{-3}$ & 15 \\
\hline Allograft rejection & $6.42 \mathrm{E}^{-3}$ & 8 \\
\hline Hematopoietic cell lineage & $6.74 \mathrm{E}^{-3}$ & 13 \\
\hline Cholinergic synapse & $7.69 \mathrm{E}^{-3}$ & 14 \\
\hline Formyl peptide receptors that bind formyl peptides and other ligands & $9.54 \mathrm{E}^{-3}$ & 4 \\
\hline Cortisol synthesis and secretion & $9.62 \mathrm{E}^{-3}$ & 10 \\
\hline Graft versus host disease & $9.86 \mathrm{E}^{-3}$ & 8 \\
\hline Binding and uptake of ligands by scavenger receptors & $1.15 \mathrm{E}^{-2}$ & 8 \\
\hline Glycoprotein VI mediated activation cascade & $1.30 \mathrm{E}^{-2}$ & 9 \\
\hline Type I diabetes mellitus & $1.30 \mathrm{E}^{-2}$ & 8 \\
\hline Regulation of TLR by endogenous ligand & $1.57 \mathrm{E}^{-2}$ & 5 \\
\hline Interleukin- 4 and -13 signaling & $1.57 \mathrm{E}^{-2}$ & 13 \\
\hline Interleukin- 8 and CXCR2 mediated signaling events & $1.57 \mathrm{E}^{-2}$ & 7 \\
\hline Oxytocin signaling pathway & $1.66 \mathrm{E}^{-2}$ & 16 \\
\hline Amoebiasis & $1.80 \mathrm{E}^{-2}$ & 12 \\
\hline Cell adhesion molecules & $2.73 \mathrm{E}^{-2}$ & 15 \\
\hline Adaptive immune system & $3.05 \mathrm{E}^{-2}$ & 47 \\
\hline Scavenging by Class A receptors & $3.26 \mathrm{E}^{-2}$ & 5 \\
\hline Natural killer cell mediated cytotoxicity & $3.29 \mathrm{E}^{-2}$ & 14 \\
\hline Collagen formation & $3.40 \mathrm{E}^{-2}$ & 11 \\
\hline Dilated cardiomyopathy & $3.40 \mathrm{E}^{-2}$ & 11 \\
\hline Antigen processing and presentation & $3.58 \mathrm{E}^{-2}$ & 10 \\
\hline Signaling pathways & $3.85 \mathrm{E}^{-2}$ & 130 \\
\hline Interferon- $\gamma$ signaling & $3.88 \mathrm{E}^{-2}$ & 11 \\
\hline Elastic fiber formation & $4.08 \mathrm{E}^{-2}$ & 7 \\
\hline Rho GTPase cycle & $4.17 \mathrm{E}^{-2}$ & 14 \\
\hline Integrin cell surface interactions & $4.17 \mathrm{E}^{-2}$ & 9 \\
\hline Classical antibody mediated complement activation & $4.51 \mathrm{E}^{-2}$ & 3 \\
\hline
\end{tabular}

CapeOX, capecitabine and oxaliplatin; GPCR, G protein coupled receptor; CXCR, CXC chemokine receptor; TLR, Toll-like receptor. 

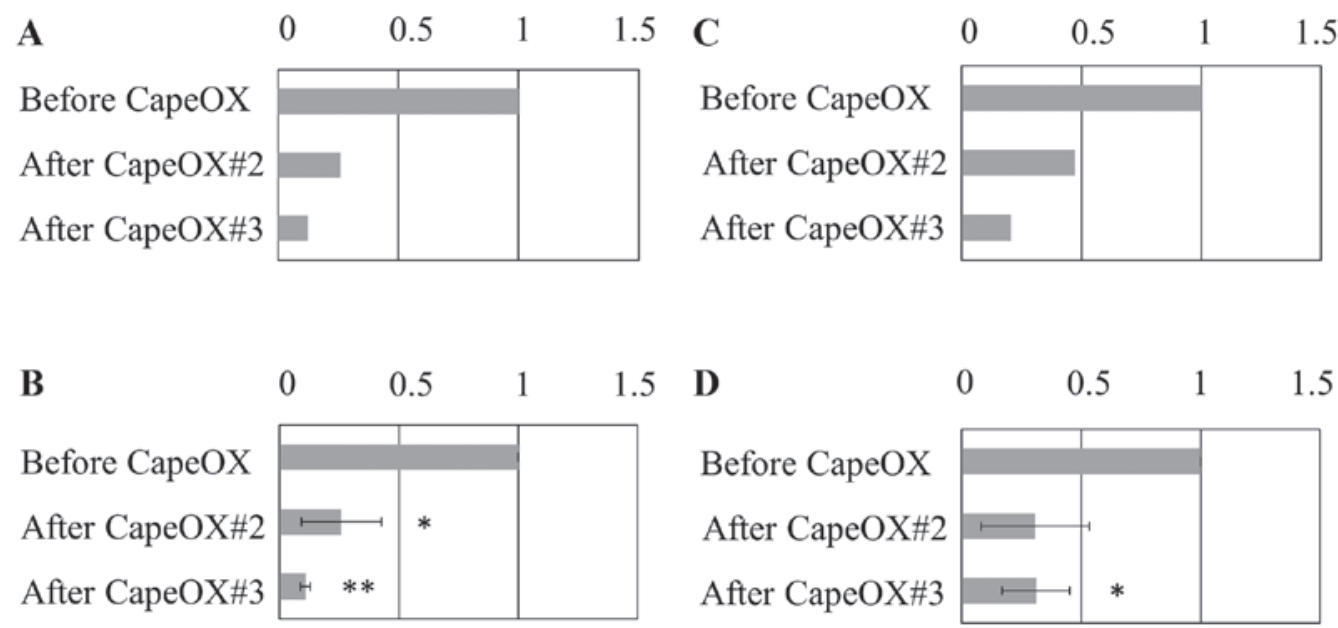

Figure 2. Expression analysis of DEFB4A (A and B) and PCDH2O (C and D) performed using a microarray (A and C) and RT-qPCR (B and D). The expression levels of each gene in ascitic cells obtained before chemotherapy were set to one, and the relative expression levels are shown for cells obtained after two cycles (CapeOX\#2) and three cycles (CapeOX\#3) of CapeOX. ${ }^{*} \mathrm{P}<0.05 ;{ }^{* *} \mathrm{P}<0.001$ vs. before CapeOX. CapeOX, capecitabine and oxaliplatin; DEFB4A, defensin $\beta 4 \mathrm{~A}$; PCDH20, protocadherin-20.

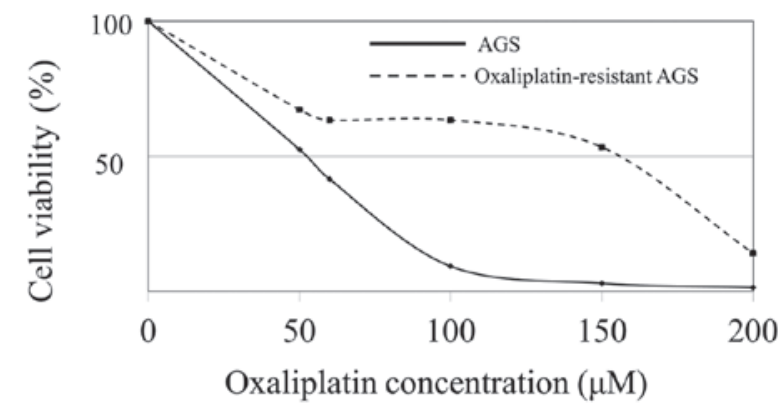

Figure 3. Oxaliplatin resistance of parent AGS cells and cells cultured in oxaliplatin-containing medium. To measure viability, cells were cultured in 96-well microplates for $24 \mathrm{~h}$, and subsequently exposed to various concentrations of oxaliplatin for $48 \mathrm{~h}$.

Establishment of oxaliplatin-resistant AGS cells. Subsequently, three oxaliplatin-resistant cell lines were established. To confirm that the gastric cancer cells cultured in oxaliplatin had acquired drug resistance, $\mathrm{IC}_{50}$ values were measured. The $\mathrm{IC}_{50}$ value for oxaliplatin was $52 \mu \mathrm{M}$ in parent AGS cells, whereas those of the three oxaliplatin-resistant AGS cells were 154, 168 and $209 \mu \mathrm{M}$ (Fig. 3).

Comparison of gene expression and DNA methylation profiles between cells collected from ascites and gastric cancer cell lines. The expression profiles of 18,185 genes were analyzed by microarray and compared among ascitic fluid cells collected before and after CapeOX treatment, drug-resistant AGS cells, and decitabine-treated AGS cells. The expression profiles of 29 genes showed that their expression levels were higher, whereas those of 368 genes revealed that their expression levels were lower, in CapeOX-resistant cells and three oxaliplatin-resistant cell lines compared with cells collected before CapeOX treatment (Table SIV). The pathways associated with these 397 genes with altered expression profiles in CapeOX-resistant cells and oxaliplatin-resistant cells were connected with interferon signaling (Tables III and SV).
The expression profiles of 107 genes showed that they were expressed at higher levels, and 55 genes were expressed at lower levels, in CapeOX-resistant cells and fluorouracil-resistant AGS cells compared with the cells obtained prior to CapeOX treatment (Table SVI). Pathway analysis of these 162 genes, however, revealed that no particular pathway was enriched.

The expression profiles of 23 genes showed that they were expressed at higher levels in CapeOX-resistant ascitic cells, fluorouracil-resistant AGS cells and oxaliplatin-resistant AGS cells compared with cells obtained prior to CapeOX. These genes included telomerase reverse transcriptase (TERT), apolipoprotein $\mathrm{C} 1$ (APOC1) and serine/threonine/tyrosine kinase 1 (STYK1). On the other hand, 18 genes, including DEFB4A, were expressed at higher levels in the pre-CapeOX cells (Table IV).

The number of genes for which methylation levels were different between drug-resistant AGS cells and parent AGS cells was analyzed. We found that, in fluorouracil-resistant AGS cells and oxaliplatin-resistant AGS cells, 1,483 and 1,579 genes, respectively, were hypermethylated, whereas 525 and 350 genes, respectively, were hypomethylated (Tables V and SVII). In contrast, only 25 and 4 genes were hyper- and hypomethylated, respectively, in cells obtained after CapeOX treatment compared with their methylation status in cells obtained prior to CapeOX. Out of the 25 genes that were hypermethylated in ascites cells collected after CapeOX, three genes [amyloid- $\beta$ precursor protein binding family B member 2 (APBB2), prostate androgen-regulated transcript 1 (PART1), and complement C1q subcomponent subunit A (C1QA)] were hypermethylated in fluorouracil-resistant AGS cells, and three other genes [serine/threonine-protein kinase 38-like (STK38L), kallikrein-related peptidase 11 (KLK11), and mesothelin (MSLN)] were hypermethylated in oxaliplatin-resistant AGS cells. In addition, PDCD1LG2 was hypomethylated in ascitic cells obtained after CapeOX and fluorouracil-resistant AGS cells.

Genes with increased expression levels in AGS cells after decitabine treatment. Out of 18,185 analyzed genes, 1,902 genes were expressed at $>2$-fold higher levels in AGS cells treated 
Table III. Pathways associated with genes that showed common changes between CapeOX resistant and oxaliplatin resistant cells.

\begin{tabular}{lrr}
\hline Pathway & P-value & No. of genes \\
\hline Interferon- $\alpha / \beta$ signaling & $4.00 \mathrm{E}^{-7}$ & $1.49 \mathrm{E}^{-3}$ \\
Interferon- $\gamma$ signaling & $1.55 \mathrm{E}^{-3}$ & 17 \\
Endosomal/vacuolar pathway & $2.69 \mathrm{E}^{-3}$ & 5 \\
Interferon signaling & $2.71 \mathrm{E}^{-3}$ & 11 \\
Human papillomavirus infection & $8.03 \mathrm{E}^{-3}$ & 21 \\
Immune system & $8.95 \mathrm{E}^{-3}$ & 69 \\
Signaling by GPCRs & $1.02 \mathrm{E}^{-2}$ \\
Cytokine signaling in the immune system & $3.05 \mathrm{E}^{-2}$ \\
Olfactory signaling pathway & $3.05 \mathrm{E}^{-2}$ \\
GPCR downstream signaling & $3.05 \mathrm{E}^{-2}$ \\
Antigen presentation: folding, assembly and peptide loading class I MHC & $3.05 \mathrm{E}^{-2}$ & 47 \\
Allograft rejection & $4.33 \mathrm{E}^{-2}$ \\
G (s) signaling events & $4.33 \mathrm{E}^{-2}$ \\
Antigen processing and presentation & 32 \\
\hline
\end{tabular}

CapeOX, capecitabine and oxaliplatin; GPCR, G protein coupled receptor.

Table IV. Genes with commonly altered expression levels in CapeOX resistant ascitic cells, fluorouracil resistant AGS cells and oxaliplatin resistant AGS cells.

\begin{tabular}{ll}
\hline Commonly increased & Commonly decreased \\
\hline ABCA12 & ANXA13 \\
APOBEC4 & CASQ1 \\
APOC1 & DEFB4A \\
ARHGDIB & ELANE \\
C19orf77 & FAIM3 \\
EPN3 & FBLN7 \\
FAM105A & HSD3B2 \\
FGD3 & IL10 \\
FGFBP1 & MYH6 \\
FOLR3 & OR1L3 \\
HLA DMA & OR7A17 \\
IFI30 & PLA2G4D \\
IGF2BP3 & PLA2G7 \\
IRF8 & S100A7A \\
LILRA4 & SLC34A2 \\
MFSD2A & SNORA22 \\
MGAT3 & TLR9 \\
NPPB & TMC5 \\
SLCO2B1 & \\
STXBP6 & \\
STYK1 & \\
TERT & \\
VTN & \\
\hline & \\
\hline
\end{tabular}

CapeOX, capecitabine and oxaliplatin.

with a demethylating agent, decitabine. Among these genes, 61 exhibited lower methylation levels, and 35 (57\%) were expressed at higher levels in fluorouracil-resistant AGS cells. By contrast, among the 16,283 genes that were not significantly increased by decitabine, only 77 of the 464 genes (17\%) that were expressed at higher levels in fluorouracil-resistant AGS cells also exhibited lower methylation levels.

\section{Discussion}

Among the four genes that were expressed at lower levels and exhibited higher levels of DNA methylation after CapeOX, WISP1 has previously been reported to be associated with WNT1 signaling and carcinogenesis (7). However, the significance of the changes in methylation of these four genes remains unclear. In addition, some discrepancies were identified between the expression and methylation profiles. For example, C1QA showed increased expression and hypermethylation after CapeOX treatment (data not shown). In the case of such genes, mechanisms other than DNA methylation could serve roles in regulating the expression.

The expression levels of 23 genes, including TERT, APOC1 and STYK1, were higher in CapeOX-resistant ascitic cells, fluorouracil-resistant AGS cells and oxaliplatin-resistant AGS cells compared with ascitic cells obtained before CapeOX treatment. TERT maintains telomere ends due to the addition of the telomere repeat, TTAGGG. Thus, telomerase expression has a role in cellular senescence since it is normally repressed in postnatal somatic cells, resulting in the progressive shortening of telomeres (8). APOC1 is a member of the apolipoprotein $\mathrm{C} 1$ family, and has been reported to be upregulated in gastric cancer (9); it has also been identified as a prognostic marker in lung cancer, as it is highly expressed in late-stage lung cancer (10). Our research group previously reported on gene alterations that were detected in gastric cancer treated with an oral fluoropyrimidine $\mathrm{S}-1$ plus cisplatin (SP) regimen; expression levels of the identified genes were altered after chemotherapy, and in fluorouracil-resistant cells and cisplatin-resistant cells (4). In the present study, it 
Table V. Genes with altered methylation levels comparing between ascetic fluid cancer cells collected before and after CapeOX treatment.

\begin{tabular}{|c|c|c|c|}
\hline Gene & $\begin{array}{l}\text { Increase in } \\
\text { methylation } \\
\text { levels after } \\
\text { CapeOX }\end{array}$ & Gene & $\begin{array}{c}\text { Decrease in } \\
\text { methylation } \\
\text { levels after } \\
\text { CapeOX }\end{array}$ \\
\hline FAM193A & 0.228 & PI3 & 0.400 \\
\hline FMO1 & 0.165 & PDCD1LG2 & 0.258 \\
\hline GPR75 & 0.142 & IFI44L & 0.160 \\
\hline NPHS1 & 0.140 & SFTA1P & 0.106 \\
\hline SEMA3B & 0.139 & & \\
\hline ANGPTL7 & 0.139 & & \\
\hline YPEL3 & 0.136 & & \\
\hline WISP1 & 0.126 & & \\
\hline STK38L & 0.126 & & \\
\hline ARHGEF25 & 0.118 & & \\
\hline SLC44A2 & 0.117 & & \\
\hline MAB21L2 & 0.114 & & \\
\hline OR10J1 & 0.111 & & \\
\hline KLK11 & 0.110 & & \\
\hline FXYD1 & 0.108 & & \\
\hline GPR110 & 0.106 & & \\
\hline APBB2 & 0.106 & & \\
\hline AREG & 0.106 & & \\
\hline ELF3 & 0.105 & & \\
\hline PART1 & 0.105 & & \\
\hline LOC285419 & 0.102 & & \\
\hline MSLN & 0.102 & & \\
\hline ECEL1P2 & 0.102 & & \\
\hline C1QA & 0.101 & & \\
\hline PYGM & 0.100 & & \\
\hline
\end{tabular}

CapeOX, capecitabine and oxaliplatin.

was observed that, among those previously identified genes, APOC1 exhibited an elevated level of expression following CapeOX treatment, after the SP regimen, and in drug-resistant cells. STYK1 is a receptor protein tyrosine kinase that serves important roles in a diverse array of cellular and developmental processes, including cell proliferation, differentiation, and survival (11). However, the 19 genes that were expressed at lower levels included DEFB4A, which has previously been reported to be a potential tumor suppressor gene (12).

Among the genes in which methylation was altered after CapeOX, PART1 is induced by androgens in prostate adenocarcinoma cells, and its expression has been reported to be associated with a poor prognosis in lung cancer (13); C1QA acts in the tumor microenvironment as a cancer-promoting factor independent of complement activation (14); KLK11 has been implicated in carcinogenesis, could potentially be a novel cancer biomarker, and is expressed at lower levels in gastric cancer, for which it might serve as a novel independent prognostic marker (15); and PDCD1LG2 expression has been reported to be inversely associated with a Crohn-like lymphoid reaction in colorectal cancer, suggesting a possible role for PDCD1LG2-expressing tumor cells in inhibiting the development of tertiary lymphoid tissues during colorectal carcinogenesis (16). No association has been found between the methylation levels of these genes and cancer, and the significance of the altered methylation levels that were observed in ascitic cells remains to be determined. However, the MSLN promoter was reported to be hypomethylated in malignant mesothelioma, although its expression was not associated with the methylation status of the promoter (17).

Among pathways potentially associated with resistance to CapeOX, GPCRs and their ligands have been reported to be involved in cancer initiation and progression, including aberrant cell proliferation, invasion, metastasis, migration, adhesion and angiogenesis, and they are considered as one of the most useful therapeutic targets for treating cancer (18). Pathways associated with 'immune system' include chemokines and interleukins, and these have also been suggested to be connected with CapeOX resistance. These results indicate that sensitivity to chemotherapeutic agents could be associated with immunological responses.

In AGS cells, the majority of genes that showed lower methylation levels following treatment with decitabine were expressed at higher levels in fluorouracil-resistant AGS cells, suggesting that the biological changes induced by chemotherapy drugs are associated with epigenetic mechanisms. However, due to the lack of success of experiments in which cancer cells from ascetic fluid were treated with a demethylating agent, the influence of epigenetics on peritoneal cancer cells remains unclear. In addition, the number of genes that showed abnormalities in DNA methylation between ascitic fluid cells collected before and after chemotherapy was lower compared with the number of genes that showed similar differences between drug-resistant and parental AGS cells. These results suggested that the drug-resistance mechanisms that act in the short term are not strongly associated with the DNA methylation changes observed in cultured cell lines.

Certain limitations of the present study should be mentioned. First, ascitic fluids from only one patient were collected and used for microarray, which is a severe limitation. Samples obtained from many more patients need to be analyzed to identify biomarkers that are more generally applicable in a clinical setting. Secondly, although it is the opinion of the present authors that the cultured cells from ascites were gastric cancer cells, the possibility of contamination with non-cancer cells cannot be excluded. Thirdly, cancer cells cultured to exclude normal cells might exhibit biological features different from cancer cells that have not been cultured in this manner. Fourthly, the cultured ascitic cells did not exhibit as much proliferative activity as other cultured cells, such as AGS cells, and were not viable enough to evaluate either drug-sensitivity or the effects of treatment with decitabine. Fifthly, AGS cells were used as the only gastric cancer cell line; using multiple cell lines with different biological features would provide more precise findings, that could be applied more universally. However, in conclusion and considering the findings of the present study as a whole, the repetitive collection of gastric cancer cells from ascitic fluids obtained before and after chemotherapy has been demonstrated to be useful in terms of analyzing the biological alterations associated with drug-resistance mechanisms. 


\section{Acknowledgements}

We thank Ms Chie Moriyama for providing technical support. Note that this paper was presented in part at the Gastrointestinal Cancers Symposium, San Francisco, CA, USA, on Jan 21-23, 2016 (J Clin Oncol: 34(4) S abstract 60, Feb 1, 2016).

\section{Funding}

This work was supported by grants from the Ministry of Education, Culture, Sports, Science and Technology of Japan (grant no. 26460936) and the Nitto Foundation.

\section{Availability of data and materials}

All data generated or analyzed during this study are included in this published article.

\section{Authors' contributions}

$\mathrm{OM}$ and YA designed the study and conducted the experiments; AM, KF, RM and YH made substantial contributions during the course of chemotherapy; and YA reviewed and edited the manuscript. All authors read and approved the final manuscript.

\section{Ethics approval and consent to participate}

The present study was approved by the Ethics Committee of Nagoya University Hospital (Nagoya, Japan). Informed consent was obtained from all patients.

\section{Patient consent for publication}

All participants provided written informed consent for the whole study.

\section{Competing interests}

YA has received research funding from Chugai Pharmaceutical Co., Ltd. and Yakult Honsya Co., Ltd.

\section{Authors' information}

ORCID numbers: OM (0000-0003-4700-6541), AM (0000-0001-9667-404X), KF (0000-0003-0980-9095), RM (0000-0001-7172-4602), YH (0000-0001-9639-7425), YA (0000-0002-6849-2297).

\section{References}

1. Koizumi W, Narahara H, Hara T, Takagane A, Akiya T, Takagi M, Miyashita K, Nishizaki T, Kobayashi O, Takiyama W, et al: S-1 plus cisplatin versus S-1 alone for first-line treatment of advanced gastric cancer (SPIRITS trial): A phase III trial. Lancet Oncol 9: 215-221, 2008
2. Tsuburaya A, Morita S, Kodera Y, Kobayashi M, Shitara K, Yamaguchi K, Yoshikawa T, Yoshida K, Yoshino S and Sakamoto J: A randomized phase II trial to elucidate the efficacy of capecitabine plus cisplatin (XP) and S-1 plus cisplatin (SP) as a first-line treatment for advanced gastric cancer: XP ascertainment vs. SP randomized PII trial (XParTS II). BMC Cancer 12: 307, 2012.

3. Yamada Y, Higuchi K, Nishikawa K, Gotoh M, Fuse N, Sugimoto N, Nishina T, Amagai K, Chin K, Niwa Y, et al: Phase III study comparing oxaliplatin plus S-1 with cisplatin plus S-1 in chemotherapy-naïve patients with advanced gastric cancer. Ann Oncol 26: 141-148, 2015.

4. Maeda O, Ando T, Ohmiya N, Ishiguro K, Watanabe O, Miyahara R, Hibi Y, Nagai T, Yamada K and Goto H: Alteration of gene expression and DNA methylation in drug-resistant gastric cancer. Oncol Rep 31: 1883-1890, 2014.

5. Maeda $\mathrm{O}$, Ando $\mathrm{T}$, Ishiguro $\mathrm{K}$, Watanabe $\mathrm{O}$, Miyahara $\mathrm{R}$, Nakamura M, Funasaka K, Kazuhiro F, Ando Y and Goto H: Safety of repeated cell-free and concentrated ascites reinfusion therapy for malignant ascites from gastrointestinal cancer. Mol Clin Oncol 2: 1103-1106, 2014.

6. Benjamini Y and Hochberg Y: Controlling the false discovery rate: A practical and powerful approach to multiple testing. J R Stat Soc B 57: 289-300, 1995.

7. Xu L, Corcoran RB, Welsh JW, Pennica D and Levine AJ: WISP-1 is a Wnt-1- and beta-catenin-responsive oncogene. Genes Dev 14: 585-595, 2000.

8. Kyo S, Takakura M, Fujiwara T and Inoue M: Understanding and exploiting hTERT promoter regulation for diagnosis and treatment of human cancers. Cancer Sci 99: 1528-1538, 2008.

9. Yasui W, Oue N, Ito R, Kuraoka K and Nakayama H: Search for new biomarkers of gastric cancer through serial analysis of gene expression and its clinical implications. Cancer Sci 95: 385-392, 2004.

10. Ko HL, Wang YS, Fong WL, Chi MS, Chi KH and Kao SJ: Apolipoprotein C1 (APOC1) as a novel diagnostic and prognostic biomarker for lung cancer: A marker phase I trial. Thorac Cancer 5: 500-508, 2014

11. Liu L, Yu XZ, Li TS, Song LX, Chen PL, Suo TL, Li YH, Wang SD, Chen Y, Ren YM, et al: A novel protein tyrosine kinase NOK that shares homology with platelet- derived growth factor/ fibroblast growth factor receptors induces tumorigenesis and metastasis in nude mice. Cancer Res 64: 3491-3499, 2004.

12. Kamino Y, Kurashige Y, Uehara O, Sato J, Nishimura M, Yoshida K, Arakawa T, Nagayasu H, Saitoh M and Abiko Y: HBD-2 is downregulated in oral carcinoma cells by DNA hypermethylation, and increased expression of hBD-2 by DNA demethylation and gene transfection inhibits cell proliferation and invasion. Oncol Rep 32: 462-468, 2014.

13. Li M, Zhang W, Zhang S, Wang C and Lin Y: PART1 expression is associated with poor prognosis and tumor recurrence in stage I-III non-small cell lung cancer. J Cancer 8: 1795-1800, 2017.

14. Bulla R, Tripodo C, Rami D, Ling GS, Agostinis C, Guarnotta C, Zorzet S, Durigutto P, Botto M and Tedesco F: Clq acts in the tumour microenvironment as a cancer-promoting factor independently of complement activation. Nat Commun 7: 10346, 2016.

15. Wen YG, Wang Q, Zhou CZ, Yan DW, Qiu GQ, Yang C, Tang HM and Peng ZH: Identification and validation of Kallikrein-ralated peptidase 11 as a novel prognostic marker of gastric cancer based on immunohistochemistry. J Surg Oncol 104: 516-524, 2011.

16. Masugi Y, Nishihara R, Hamada T, Song M, da Silva A, Kosumi K, Gu M, Shi Y, Li W, Liu L, et al: Tumor PDCD1LG2 (PD-L2) Expression and the Lymphocytic Reaction to Colorectal Cancer. Cancer Immunol Res 5: 1046-1055, 2017.

17. Tan K, Kajino K, Momose S, Masaoka A, Sasahara K, Shiomi K, Izumi H, Abe M, Ohtsuji N, Wang T, et al: Mesothelin (MSLN) promoter is hypomethylated in malignant mesothelioma, but its expression is not associated with methylation status of the promoter. Hum Pathol 41: 1330-1338, 2010.

18. Liu Y, An S, Ward R, Yang Y, Guo XX, Li W and Xu TR: $\mathrm{G}$ protein-coupled receptors as promising cancer targets. Cancer Lett 376: 226-239, 2016. 